\title{
COMPORTAMENTO ADSORTIVO DE METAIS TRAÇO NUM ARGISSOLO, SOB CONDIÇÕES COMPETITIVAS E NÃOCOMPETITIVAS.
}

\author{
Lidia Maria de Almeida Plicas ${ }^{1}$ \\ Simone Alcina da Silva²
}

Flávio Fernando Manzini ${ }^{3}$

\begin{abstract}
RESUMO
A determinação de metais traço, em solos é uma importante ferramenta no monitoramento da poluição ambiental, para tanto, faz-se necessária a caracterização física e química do solo, a quantificação total e disponível dos metais e o estudo do comportamento da adsorção. Tal estudo é importante para estabelecer a relação entre o conteúdo total do metal e os fatores que influenciam esta relação que, por conseguinte, afeta a disponibilidade do metal para as plantas e o processo de percolação até lençóis freáticos ou a lixiviação aos cursos d'água próximos às áreas agrícolas. As isotermas de Langmuir e Freundlich foram adequadas para descrever os processos de adsorção dos metais traço analisados. Observou-se que o metal traço $\mathrm{Cd}$ foi o que apresentou os maiores valores de adsorção máxima $\left(Q_{\text {máx }}\right)$, o $\mathrm{Cu}$ apresentou o maior valor da constante de afinidade $\left(\mathrm{K}_{\mathrm{L}}\right)$, do modelo matemático de Langmuir. O Zn apresentou o maior valor de capacidade adsortiva $\left(\mathrm{K}_{\mathrm{f}}\right)$ e o Cd o maior valor de $1 / \mathrm{n}$ do modelo de Freundlich. Considerando o pH natural do solo, a seqüência de afinidade observada foi: $\mathrm{Cu}>\mathrm{Zn}>\mathrm{Ni}>\mathrm{Cd}$, no sistema não competitivo e a seqüência de afinidade $\mathrm{Cd}>\mathrm{Cu} \geq \mathrm{Zn}>\mathrm{Ni}$, no sistema competitivo.
\end{abstract}

PALAVRAS-CHAVE: Solo. Metais traço. Adsorção.

\section{ADSORPTION BEHAVIOR OF TRACE IN ULTISOL METALS UNDER COMPETITIVE CONDITIONS AND NONCOMPETITIVE}

\section{ABSTRACT}

\footnotetext{
${ }^{1}$ Doutora em Físico-Química, Instituto de Biociências, Letras e Ciências Exatas, UNESP-SP plicas@ibilce.unesp.br

2 Bacharel em Química Ambiental, Instituto de Biociências, Letras e Ciências Exatas, UNESP-SP si_quimica@hotmail.com

3 Doutor em Geologia Regional, Instituto de Biociências, Letras e Ciências Exatas, UNESP-SP fmanzini@ibilce.unesp.br.
} 
The determination of trace metals in soils is an important tool in monitoring environmental pollution, to this end it is necessary the soil physical and chemical characterization, the quantification total of metals and the study of adsorption behavior. Such a study it is important to establish the relationship between the total metal content and the content available and the factors that influence this relationship therefore affects the availability of metal for the plants and the process of percolation to groundwater or leaching to waterways close to agricultural areas. The isotherms of Langmuir and Freundlich were suitable to describe the processes of adsorption of trace metals analyzed. It was observed that the trace metal Cd was presented the highest values of maximum adsorption $\left(Q_{\max }\right)$, the $\mathrm{Cu}$ presented the highest value of the affinity constant $\left(\mathrm{K}_{\mathrm{L}}\right)$, the mathematical model of Langmuir. The $\mathrm{Zn}$ presented the greatest value of adsortiva capacity $\left(\mathrm{K}_{\mathrm{f}}\right)$ and the $\mathrm{Cd}$ the greatest value of $1 / \mathrm{n}$. Considering the natural $\mathrm{pH}$ of the soil, the affinity observed was: $\mathrm{Cu}>\mathrm{Zn}>\mathrm{Ni}>\mathrm{Cd}$ in non-competitive system and the affinity $\mathrm{Cd}>\mathrm{Cu} \geq \mathrm{Zn}>\mathrm{Ni}$, in the competitive system.

KEYWORDS: Soil. Trace metals. Adsorption.

\title{
ADSORCIÓN COMPORTAMIENTO DE RASTRO EN ULTISOLES METALES EN CONDICIONES COMPETITIVOS Y NO COMPETITIVOS
}

\begin{abstract}
RESUMEN
La determinación de metales traza en los suelos es una herramienta importante en la vigilancia de la contaminación ambiental, para ello, es necesario la caracterización física y química de suelos, la cuantificación total de metales y el estudio del comportamiento de adsorción. Este estudio es importante establecer la relación entre el contenido total de metal y los contenidos disponibles y los factores que influyen en esta relación, por tanto, afecta la disponibilidad de metal para las plantas y el proceso de filtración a las aguas subterráneas o lixiviación a vías navegables cerca de zonas agrícolas. Las isotermas de Langmuir y Freundlich fueron adecuadas describir los procesos de adsorción de metales traza analizados. Se observó que el metal traza Cd se presentó los valores más altos de la máxima adsorción $\left(\mathrm{Q}_{\max }\right)$, el Cu presentó el mayor valor de la constante de afinidad $\left(\mathrm{K}_{\mathrm{L}}\right)$, delo modelo matemático de Langmuir. El Zn presentó el mayor valor de capacidad adsortiva $\left(\mathrm{K}_{\mathrm{f}}\right)$ y el $\mathrm{Cd}$, el valor mayor de 1/n. Considerando el $\mathrm{pH}$ natural del suelo, la afinidad observada fue: $\mathrm{Cu}>\mathrm{Zn}>\mathrm{Ni}>\mathrm{Cd}$ en sistema no competitivo y la afinidad $\mathrm{Cd}>\mathrm{Cu} \geq \mathrm{Zn}>\mathrm{Ni}$, en el sistema competitivo.
\end{abstract}

PALABRAS-CLAVE:Suelo. Metales traza. Adsorción.

\section{INTRODUÇÃO}

Manter-se a boa qualidade do meio ambiente é fundamental para a preservação da vida. Grande parte dos rios, lagos e represas vêm sendo contaminados pela descarga inadequada de poluentes; solos vêm tornando-se alvos de depósitos de resíduos urbanos e industriais. $O$ solo, quando manejado de forma descuidada, pode ser uma fonte importante de poluição. A utilização, de maneira não apropriada, de defensivos agrícolas, de águas de irrigação de baixa qualidade e a disposição indiscriminada de resíduos industriais ou domésticos pode provocar o 
acúmulo de substâncias impróprias que podem ser tóxicas para plantas e podem entrar na cadeia alimentar, afetando o ser humano.

Em função das características do solo, a água infiltra-se e atravessa os diversos substratos horizontais. A água subterrânea, propriamente dita, encontra-se nas zonas saturadas, onde os poros, fraturas ou espaços vazios da matriz sólida estão completamente preenchidos por água. Como água subterrânea, água superficial e solo, fazem parte do mesmo contexto, a migração dos poluentes através do solo, para as águas superficiais e subterrâneas, constitui uma ameaça para a qualidade dos recursos hídricos.

Defensivos agrícolas, também conhecidos como agroquímicos são produtos empregados para evitar ou reduzir os danos econômicos causados por pragas, doenças e ervas invasoras na área agrícola. Sua utilização na agricultura moderna é bastante freqüente e segura, mas pode provocar efeitos nocivos à saúde e ao meio ambiente quando aplicados inadequadamente. (ARACRUZ, 2007).

Dentre os agroquímicos utilizados nas atividades agrícolas, os fertilizantes e alguns fungicidas, são aqueles com maior potencial de inserção de elementostraço nos solos.Segundo Andrade (2006), os elementos metálicos presentes nos solos são de origem litogênica ou antropogênica, a fração litogênica é liberada pelas rochas através de processos intempéricos, sendo que estes elementos participarão de diversos ciclos tanto bióticos como abióticos até ocorrer deposição final nos oceanos ou sedimentos.

As rochas fosfáticas usadas para a produção dos fertilizantes constituem também uma fonte de contaminação com elementos-traço em solos agrícolas (LAVADO et al., 2001), pois podem apresentar em sua composição íons metálicos deCd, $\mathrm{Pb}, \mathrm{As}, \mathrm{Hg}$ e Se além de $\mathrm{Cr}, \mathrm{U}, \mathrm{V}$ entre outros. (UNIVERSIA, 2006).

Os metais, em solos, normalmente, apresentam-se em concentrações muito pequenas, associados a outros elementos formando minerais rochosos, por isso, quando determinados em altas concentrações, é indicativo do resultado de alguma atividade antrópica. Quando lançados no solo podem ser absorvidos pelos tecidos animais e vegetais podendo acumular-se em todos os organismos que constituem a cadeia alimentar do homem. (GUARACHO, 2005). 
Devido à sua heterogeneidade natural, quando o solo está exposto a substâncias externas a sua origem ocorrem processos de interação físicos e químicos dos mais diversos, relacionados com a perda ou ganho de massa do contaminante para o solo, e que também são regulados por diversas variáveis como pH, concentração das substâncias, granulometria dos minerais, composição dos minerais, matéria orgânica, permeabilidade, ente outras. (ALLOWAY, 1995).

Os processos químicos que podem ocorrer entre o solo e o contaminante são reações do tipo: adsorção-desorção, ácido-base, dissolução-precipitação, oxidaçãoredução, processos biológicos e complexação. A transferência real de contaminantes da fase líquida para a fase sólida acontece nas reações de adsorção-desorção e precipitação-dissolução. (MACHADO et al., 2003).

Sposito (1989) define a adsorção como um processo que leva ao acúmulo de matéria na interface entre uma fase sólida e líquida. A superfície sólida é o adsorvente e o adsorbato é a molécula ou íon da solução que pode ser adsorvido.

De acordo com Drever (1997 apud DEMUELENAERE, 2004), o mecanismo de adsorção pode ser dividido em: adsorção física, onde a atração ocorre através de forças de Van der Waals, relativamente fracas; adsorção eletrostática, onde os íons na solução são atraídos pela superfície de carga elétrica oposta; e adsorção química entre as moléculas do soluto em um ou mais átomos na superfície do sólido. Para se entender a migração dos metais traço nos solos e na água subterrânea, é preciso modelar quantitativamente os processos de adsorção. A forma que relaciona a concentração de espécies adsorvidas nos sólidos e a concentração na solução é geralmente referida como isoterma.

Para se quantificar este processo, Freeze(1979) salienta que se deve quantificar o coeficiente de distribuição $\left(K_{d}\right)$, baixos valores de $K_{d}$ indicam que a maior parte do metal presente no sistema permanece em solução e, portanto, disponível para transporte ou outros processos químicos ou para a absorção das plantas. Altos valores refletem grande afinidade dos componentes sólidos do solo pelo elemento (ANDERSON, 1988).

A mobilidade do poluente num fluxo subsuperficial também pode ser indicada por este coeficiente. Conforme USEPA (1999), o coeficiente de distribuição, Kd, é um dos parâmetros mais importantes usados para se estimar o potencial de migração de 
contaminantes presentes em soluções líquidas, em contato com sólidos. Tal coeficiente é determinado pelo cociente da concentração de metal adsorvido ( $\mathrm{mg} \mathrm{kg}^{-1}$ ) pela concentração do metal em equilíbrio ( $\left.\mathrm{mg} \mathrm{L}^{-1}\right)$, logo, as unidades de $\mathrm{Kd}_{\mathrm{d}}$ são $\mathrm{L} \mathrm{kg}^{-1}$.

O modelo de isoterma de Langmuir pressupõe um equilíbrio relacionando a quantidade de soluto adsorvido em uma superfície com a concentração do soluto na solução. Supõe, ainda, que a superfície do sólido é coberta por um grande número de sítios, sendo que cada sítio pode ser ocupado por uma molécula adsorvida. Os sítios são todos equivalentes e considera-se que as moléculas adsorvidas não interagem umas com as outras nem saltam de um sítio para outro. A adsorção completa-se quando todos os sítios forem ocupados, correspondendo a uma monocamada de adsorvato. Para esse modelo a adsorção é constante, ou seja, a energia do adsorvido é a mesma em qualquer ponto da superfície e independente da presença de moléculas adsorvidas na vizinhança, sendo todos os pontos equivalentes. Esse modelo é descrito pela equação 1:

$$
\mathrm{C}_{\text {eq }} / \mathrm{C}_{\text {ads }}=1 /\left(\mathrm{K}_{\mathrm{L}} \mathrm{Qmáx}\right)+\left(\mathrm{C}_{\mathrm{eq}} \mathrm{Qmáx}\right)
$$

Em que $\mathbf{C}_{\text {eq }}$ é a concentração do soluto na solução de equilíbrio ( $\mathrm{mg} \mathrm{L}^{-1}$ ), $\mathbf{C}_{\text {adsé }}$ a quantidade de soluto adsorvido por massa de adsorvente ( $\mathrm{mg} \mathrm{kg}^{-1}$ ), $\mathrm{K}_{\mathrm{L}}$ é a constante de afinidade relacionada à energia de ligação soluto-superfície adsorvente, e Qmáx é o limite superior de $C_{a d s}$, que mostra a capacidade máxima de adsorção do soluto $\left(\mathrm{mg} \mathrm{kg}^{-1}\right)$.

A isoterma de Freundlich é representada por uma equação empírica que pode ser aplicada a sistemas não ideais, em superfícies heterogêneas e adsorção em multicamadas (COONEY, 1999; RUTHVEN, 1984). O modelo admite que a energia de adsorção decrescelogaritmicamente à medida que a superfície vai se tornando coberta pelo adsorvato (SOARES, 2004). Nesse modelo, a relação entre a concentração adsorvida e a concentração de equilíbrio pode ser representada pela equação 2:

$$
\log \mathrm{C}_{\mathrm{ads}}=\log \mathrm{K}_{\mathrm{f}}+(1 / \mathrm{n}) \log \mathrm{C}_{\mathrm{eq}}
$$


Em que $\mathbf{K}_{\mathbf{f}}$ é o coeficiente de Freundlich, indica a capacidade do solo em reter o soluto $\left(\mathrm{mg} \mathrm{g}^{-1}\right)$ e $\mathbf{1 / n}$ indica a afinidade do solo pelo soluto, o qual é um parâmetro adimensional. (SOARES, 2004).

\section{OBJETIVOS}

Considerando os fatores ambientais que podem afetar diretamente a mobilidade e disponibilidade dos metais traço pretende-se realizar: (i) o estudo de adsorção de metais traço em amostra de um argissolo de São José do Rio Preto, em sistema competitivo e nãocompetitivo e (ii) estabelecer as sequências de afinidade dos íons metálicos, com base nos valores de capacidade máxima de adsorção dos metais, estimados por meio do modelo de Langmuir, em sistema competitivo e nãocompetitivo

\section{MATERIAL E MÉTODOS}

\subsection{Materiais}

Utilizou-se vidrarias comuns de laboratórios, erlenmeyers de polipropileno, conjunto de filtração a vácuo, balança analítica OHAUS, balança semi-analítica GEHAKA, pHmetro (Digimed DM 22), mesa agitadora (LogenScientific), centrífuga (LogenScientific), membrana filtrante $0,45 \mu \mathrm{m}$ (Millipore) e espectrômetro de absorção atômica por chama (Varian, modelo SpectrAA 50B).Foram utilizados reagentes de grau P.A., e soluções padrões SpecSol, de $1000 \mathrm{mg} \mathrm{L}^{-1}$ dos íons metálicos, rastreadas.

\subsection{Coleta e Preparo da Amostra}

As amostras de solo estéril utilizadas foram coletadas na cidade de São José do Rio Preto, Estado de São Paulo. Dividiu-se a extensão da área uniformemente, para a retirada de amostras, a área foi percorrida em ziguezague, retirando-se, com um trado,amostras de 15 a 20 pontos diferentes. Após a limpeza da superfície do solo, removendo detritos, as amostras foram retiradas da camada superficial, até a profundidade de $20 \mathrm{~cm}$, foram acondicionadas em sacos plásticos devidamente etiquetados. No laboratório formou-se uma amostra composta, a qual foi seca ao ar, 
depois homogeneizada, passando-se em peneira de $2 \mathrm{~mm}$ de abertura, resultando na Terra Fina Seca ao Ar (TFSA).

\subsection{Métodos}

- Determinação dos metais, teor total, no solo, segundo método oficial 3050B que consiste resumidamente em extração, a quente, com $\mathrm{HNO}_{3}$ concentrado seguido de análise por espectrometria de absorção atômica por atomização em chama (EAA). (USEPA, 1998)

- Análise da adsorção em sistema não-competitivo. (LAIR, et al., 2006)

- Análise da adsorção em sistema competitivo. (ARAÚJO et al., 2009)

Em 2,0 g de TFSA foram adicionados $20 \mathrm{~mL}$ de solução de $\mathrm{NaNO}_{3} 0,01 \mathrm{~mol} \mathrm{~L}^{-1}$ (relação 1:10) contendo diferentes concentrações iniciais dos metais na forma, preferencialmente, de sais de nitrato que variaram entre 0,017 a 1,275 mmol L-1 $\mathrm{O}$ íon nitrato foi escolhido como eletrólito suporte devido à sua menor habilidade em complexar cátions metálicos.Cada batelada foi agitada por 1 hora em agitador horizontal a 100 osc $\mathrm{min}^{-1}$, a $25 \stackrel{\circ}{\circ} \mathrm{C}$ (FONTES, 2003). Após agitação, as amostras foram filtradas em papel de filtro qualitativo e posteriormente em membrana de nitrato de celulose de $0,45 \mu \mathrm{m}$. As concentrações do metal em equilíbrio foram determinadaspor Espectroscopia de Absorção Atômica por Chama.

Foram elaborados gráficos das quantidades adsorvidas, Cads, em função das concentrações de equilíbrio, $\mathrm{C}_{\text {eq, }}$ em cada temperatura de análise. As isotermas linearizadas de adsorção de Langmuir e Freundlich foram testadas a fim de verificar qual delas melhor representa o processo de adsorção para posterior cálculo da constante de adsorção.

\section{RESULTADOS E DISCUSSÕES}

\subsection{Análises granulométrica e químicas.}

Os resultados relevantes, das análises granulométrica e químicas, para o presente trabalho encontram-se dispostos na Tabela 1. 
Tabela 1. Resultados da caracterização granulométrica e química do solo amostrado

\begin{tabular}{ccccccc}
\hline & Granulometria & & \multicolumn{2}{c}{ pH } & M. O. & CTC \\
\hline Areia $\%$ & Silte $\%$ & Argila $\%$ & $\mathrm{H}_{2} \mathrm{O}$ & $\mathrm{CaCl}_{2}$ & $\mathrm{~g} \mathrm{dm}^{-3}$ & $\mathrm{mmol}_{c} \mathrm{dm}^{-3}$ \\
\hline 84,22 & 13,80 & 1,98 & 5,75 & 5,05 & 24,8 & 62,0 \\
\hline
\end{tabular}

Fonte: Autores, 2013.

O solo, do tipo argissolo, é considerado arenoso por apresentar quantidade de argila inferior a $15 \%$, apresenta acidez baixa. $\mathrm{O} \mathrm{pH}$ em água normalmente, possui valores maiores entre 0,3 a 1,2 unidades em relação a valores de $\mathrm{pH}$ em $\mathrm{CaCl}_{2}$. $\mathrm{A}$ amostra de solo apresentou variação dentro do esperado. (TOMÉ JR.1997).

Oteor de matéria orgânica determinadopode ser considerado baixo, conforme Tomé Jr. (1997). A diminuição da matéria orgânica no solo pode representar um aumento na densidade aparente do solo, dificultando a percolação da água, aumentando o escoamento superficial e resultando em maiores taxas de erosão.

A porcentagem da capacidade de troca catiônica é de grande importância para avaliação da fertilidade do solo, retenção de nutrientes e de água. A CTC foi calculada a partir da soma dos cátions $\mathrm{Na}^{+}, \mathrm{K}^{+}, \mathrm{Mg}^{+2}, \mathrm{Ca}^{+2}$ e acidez potencial $\left(\mathrm{H}^{+}+\mathrm{Al}^{+3}\right)$. O valor da CTC pode ser considerado médio. (RAIJ, 2001).

\subsection{Determinação das concentrações totais dos metais traço no solo.}

Os metais traço analisados foram Cádmio, Níquel, Cobre e Zinco, embora estes dois últimos não sejam considerados metais traço, quando acima de valores regulados pela CONAMA 409/2009, podem ser considerados tóxicos. Os resultados das concentrações totais destes íons metálicos estão apresentados na Tabela 2.

Tabela 2. Concentração total dos metais traço analisados no solo amostrado

\begin{tabular}{c|c}
\hline Metal traço & Concentração média em solo $\left(\mathbf{m g ~ k g} \mathbf{~}^{-1}\right)$ \\
\hline Cádmio & 9,5 \\
Níquel & 7,5 \\
Cobre & 11,1 \\
Zinco & 30,3 \\
\hline
\end{tabular}

Fonte: Autores, 2014. 
Segundo a resolução Conama 420/2009, os valores obtidos não ultrapassaram os valores orientadores considerados aceitáveis. É possível inferir que estes estão associados à formação do solo, presentes na rocha parental.

\subsection{Adsorçãonão competitiva}

Todas as isotermas de adsorção dos íons de $\mathrm{Cu}^{+2}$ seguiram o tipo L (GILES, 1960), ou seja, segundo modelo de Langmuir, tem inclinação não linear e côncava em relação à abscissa. É caracterizada por um decréscimo na inclinação da curva, à medida que o número de sítios disponíveis para a adsorção diminui devido o recobrimento da superfície. Na medida em que a temperatura aumenta, as inclinações iniciais das isotermas tornam-se relativamente, menos pronunciadas.

Os dados obtidos das isotermas de adsorção, para os íons metálicos de $\mathrm{Cu}^{+2}$,foram tratados empregando-se os modelos de Langmuir e Freundlich, os valores das constantes foram calculados e estão dispostos na tabela 3.

Tabela 3. Dados das isotermas linearizadas de Langmuir e Freundlich, adsorção de íons $\mathrm{Cu}^{+2}$

\begin{tabular}{|c|c|c|c|c|c|}
\hline Isoterma & & $25,0 \stackrel{\circ}{\circ}$ & $35,0^{\circ} \mathrm{C}$ & $45,0 \stackrel{\circ}{\circ}$ & $55,0 \stackrel{\circ}{C}$ \\
\hline \multirow[t]{3}{*}{ Langmuir } & $Q_{\text {máx }} / \mathrm{mg} \mathrm{kg}^{-1}$ & 625 & 769 & 526 & 833 \\
\hline & $\mathrm{K}_{\mathrm{L}} / \mathrm{kg} \mathrm{L}^{-1}$ & 0,7 & 1,0 & 1,6 & 0,6 \\
\hline & $\mathrm{R}^{2}$ & 0,9837 & 0,9725 & 0,9925 & 0,9458 \\
\hline \multirow[t]{3}{*}{ Freundlich } & $1 / n$ & 0,48 & 0,55 & 0,61 & 0,90 \\
\hline & $\mathrm{K}_{f}$ & 175,2 & 228,5 & 241,7 & 222,6 \\
\hline & $R^{2}$ & 0,9197 & 0,9678 & 0,9082 & 0,9202 \\
\hline
\end{tabular}

Fonte: Autores, 2014.

As isotermas, para a adsorção dos íons $\mathrm{Zn}^{+2}$, apresentaram comportamento do tipo $S$, subgrupo $S 3$, e do tipo $C$ respectivamente. Têm trecho inicial de inclinação linear seguido de trecho convexo em relação à abscissa. Indica que a adsorção inicial é baixa e aumenta à medida que o número de espécies adsorvidas aumenta. $\mathrm{A}$ direção inicial da curva mostra que a adsorção torna-se mais fácil em concentrações maiores, esse tipos de curva surge quando a atração inter-molecular é moderada.Os 
foram tratados empregando-se os modelos de Langmuir e Freundlich, os valores das constantes obtidos estão dispostos na tabela 4.

Tabela 4. Dados das isotermas linearizadas de Langmuir e Freundlich, adsorção de íons $\mathbf{Z n}^{+2}$

\begin{tabular}{|c|c|c|c|c|c|}
\hline Isoterma & & $25,0^{\circ} \mathrm{C}$ & $35,0^{\circ} \mathrm{C}$ & $45,0^{\circ} \mathrm{C}$ & $55,0^{\circ} \mathrm{C}$ \\
\hline \multirow[t]{3}{*}{ Langmuir } & $Q_{\text {máx }} / \mathrm{mg} \mathrm{kg}^{-1}$ & 833 & 500 & --- & --- \\
\hline & $\mathrm{K}_{\mathrm{L}} / \mathrm{kg} \mathrm{L}^{-1}$ & 0,5 & 0,6 & --- & --- \\
\hline & $\mathrm{R}^{2}$ & 0,9626 & 0,9542 & --- & --- \\
\hline \multirow[t]{3}{*}{ Freundlich } & $1 / n$ & 0,53 & 0,44 & 1,47 & 1,16 \\
\hline & $\mathrm{K}_{\mathrm{f}}$ & 149,5 & 165,7 & 172,0 & 229,5 \\
\hline & $\mathrm{R}^{2}$ & 0,9579 & 0,9296 & 0,9806 & 0,9977 \\
\hline
\end{tabular}

Fonte: Autores, 2014.

Todas as isotermas de adsorção dos íons $\mathrm{Cd}^{+2}$ seguiram o tipo $\mathrm{L}$, o adsorvato apresenta dificuldade em encontrar sítios vazios, os quais devem estar preenchidos com íons do eletrólito suporte, $\mathrm{NaNO}_{3}$, visto que a competição entre estes e os íons de $\mathrm{Cd}^{+2}$ não é forte então, a adsorção aumenta à medida em que a concentração dos íons de $\mathrm{Cd}^{+2}$ aumenta. Os dados obtidos foram tratados e os valores das constantes de Langmuir e Freundlich foram calculados e estão dispostos na tabela 5.

Tabela 5. Dados das isotermas linearizadas de Langmuir e Freundlich, adsorção de íons $\mathrm{Cd}^{+2}$

\begin{tabular}{cccccc}
\hline Isoterma & & $\mathbf{2 5 , 0} \stackrel{\circ}{\circ}$ & $\mathbf{3 5 , 0} \stackrel{\circ}{ } \mathbf{C}$ & $\mathbf{4 5 , 0} \stackrel{\circ}{ } \mathbf{C}$ & $\mathbf{5 5 , 0} \stackrel{\circ}{ } \mathbf{C}$ \\
\hline Langmuir & $\mathrm{Q}_{\text {máx }} / \mathrm{mg} \mathrm{kg}^{-1}$ & 1429 & 2000 & 1429 & 1429 \\
& $\mathrm{~K}_{\mathrm{L}} / \mathrm{kg} \mathrm{L}^{-1}$ & 0,09 & 0,06 & 0,07 & 0,09 \\
& $\mathrm{R}^{2}$ & 0,9985 & 0,9159 & 0,9743 & 0,9831 \\
\multirow{2}{*}{ Freundlich } & $1 / \mathrm{n}$ & 0,79 & 0,25 & 0,74 & 0,70 \\
& $\mathrm{~K}_{\mathrm{f}}$ & 62,1 & 32,6 & 97,2 & 110,0 \\
& $\mathrm{R}^{2}$ & 0,9510 & 0,8512 & 0,9629 & 0,9493 \\
\hline
\end{tabular}


Fonte: Autores, 2014.

As isotermas de adsorção dos íons $\mathrm{Ni}^{+2}$ apresentaram o tipo $\mathrm{L}$, a partir de $35{ }^{\circ} \mathrm{C}$ tenderam ao comportamento do sub-grupo 3, ou seja, tendem à formação de multicamadas. A interação $\mathrm{Ni}^{+2}$-adsorvente deve ser forte levando à formação de multicamadas. Os dados obtidos, das isotermaslinearizadas de Langmuir e Freundlich, estão dispostos na tabela 6.

Tabela 6. Dados das isotermas linearizadas de Langmuir e Freundlich, adsorção de íons $\mathbf{N i}^{+2}$

\begin{tabular}{|c|c|c|c|c|c|}
\hline Isoterma & & $25,0 \stackrel{\circ}{ } \mathrm{C}$ & $35,0 \stackrel{\circ}{ }=$ & $45,0 \div C$ & $55,0 \stackrel{\circ}{C}$ \\
\hline \multirow[t]{3}{*}{ Langmuir } & $Q_{\text {máx }} / \mathrm{mg} \mathrm{kg}^{-1}$ & 435 & 667 & 714 & 714 \\
\hline & $\mathrm{K}_{\mathrm{L}} / \mathrm{kg} \mathrm{L}^{-1}$ & 0,25 & 0,10 & 0,09 & 0,11 \\
\hline & $\mathrm{R}^{2}$ & 0,9957 & 0,9754 & 0,9924 & 0,9873 \\
\hline \multirow[t]{3}{*}{ Freundlich } & $1 / n$ & 0,52 & 0,66 & 0,62 & 0,68 \\
\hline & $\mathrm{K}_{f}$ & 76,1 & 65,7 & 74,1 & 71,5 \\
\hline & $R^{2}$ & 0,9732 & 0,9702 & 0,9874 & 0,9563 \\
\hline
\end{tabular}

Fonte: Autores, 2014.

A maior porcentagem de adsorção dentre os quatro metais traço foi verificada para o $\mathrm{Zn}$, com adsorção por volta de $90 \%$, seguida de $\mathrm{Cd}$ e $\mathrm{Cu}$, com cerca de $80 \%$ de adsorção e por último o $\mathrm{Ni}$ com cerca de $60 \%$, todos calculados com relação à dose máxima adicionada de cada metal. Observou-se ainda que, na medida em que a temperatura aumenta tais porcentagens tenderam a diminuir. As características do solo que contribuem para a maior adsorção são pH, CTC e MO. Sendo assim, o Cu deveria apresentar maior retenção no solo, pois, este é retido principalmente por ácidos húmicos e fúlvicos da M.O., formando complexos estáveis, entretanto, isso não foi verificado devido, provavelmente, ao baixo $\mathrm{pH}$ do solo.

As quantidades máximas de adsorção $\left(Q_{\text {máx }}\right)$ apresentaram a seguinte ordem crescente para os metais traço: $\mathrm{Cd}>\mathrm{Zn}>\mathrm{Cu}>\mathrm{Ni}$. Observou-se ainda que, somente o

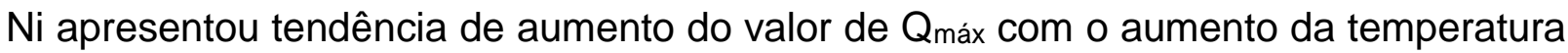
isso devido provavelmente, à tendência de formação de multicamadas. Os baixos 
valores de $\mathrm{Qmáx}_{\text {dos }}$ metais traço $\mathrm{Cu}, \mathrm{Zn}$ e Ni, relativamente ao do $\mathrm{Cd}$, podem ser devido, provavelmente, aos baixos teores de argila, de óxidos e de M.O.

Os valores de $\mathrm{K} \mathrm{L}$, que expressa a energia de ligação da adsorção, variaram cerca de 1 ordem de grandeza entre os metais analisados. Os maiores valores foram obtidos para o $\mathrm{Cu}$ e os menores para o $\mathrm{Cd}$. A ordem de afinidade encontrada, na média, foi: $\mathrm{Cu}>\mathrm{Zn}>\mathrm{Ni}>\mathrm{Cd}$. Os menores valores indicam maior potencial de mobilidade no solo e de biodisponibilidade desses metais no ambiente.

$\mathrm{O}$ parâmetro $\mathrm{K}_{\mathrm{f}}$, está relacionado com a capacidade adsortiva do solo, assim como os valores da adsorção máxima, apesar disso, não se observou a mesma tendência dos metais como daquela da adsorção máxima. A tendência observada foi a mesma para os valores de $\mathrm{K} \mathrm{L}$, ou seja: $\mathrm{Cu}>\mathrm{Zn}>\mathrm{Ni}>\mathrm{Cd}$. Moreira (2010) encontrou a mesma sequência de afinidade para a maioria dos 15 solos, do estado de São Paulo, analisados.

Quanto ao parâmetro $1 / \mathrm{n}$, todos os processos de adsorção dos metais apresentaram valores inferiores a 1, com exceção para a adsorção do $\mathrm{Zn}$ nas temperaturas de 45,0 e $55,0{ }^{\circ} \mathrm{C}$, que apresentaram valores maiores do que 1 . Variações nos valores de $1 / n$ demonstram que os íons metálicos foram adsorvidos sob diferentes níveis de energia, em concordância com o fato da distribuição dos sítios energéticos tenderem a variar com a densidade de adsorção e quanto maior o valor de $1 / \mathrm{n}$ maior a heterogeneidade dos sítios de adsorção.

A tabela 7 resume os valores de $\mathrm{K}_{d}$ determinados para os quatro metais traço no solo analisado.

Tabela 7: Dados de coeficiente de adsorção dos metais traço ao solo analisado

\begin{tabular}{ccccc}
\hline Metal traço & $\mathrm{Cu}$ & $\mathrm{Zn}$ & $\mathrm{Cd}$ & $\mathrm{Ni}$ \\
\hline $\mathrm{Kd} / \mathrm{L} \mathrm{kg}^{-1}$ & 357 & 301 & 79 & 92 \\
\hline
\end{tabular}

Fonte: Autores, 2014.

Dos resultados da tabela 7 é possível ordenar os metais traço na seguinte ordem de afinidade: $\mathrm{Cu}>\mathrm{Zn}>\mathrm{Ni}>\mathrm{Cd}$. A pesar da interpretação dos valores de $\mathrm{Kd}$ para metais ser muito mais complicada do que para compostos orgânicos, uma vez que, 
para os metais os coeficientes são afetados por diferentes condições do solo e propriedades do metal, a magnitude dos valores encaixam-se no intervalo de valores estabelecidos por Sauvé (2000), compilados de 70 estudos. A sequência de afinidade obtida entre os metais traço analisados neste trabalho difere daquela mais comum relatada em outros trabalhos, entretanto, foi a mesma encontrada a partir dos valores de $\mathrm{K}_{\mathrm{L}}$ e $\mathrm{K}_{\mathrm{f}}$, obtidos respectivamente, das isotermas de Langmuir e Freundlich. Desta sequência infere-se que os dois metais de menor afinidade são o $\mathrm{Ni} \mathrm{e} \mathrm{Cd,} \mathrm{que} \mathrm{estão}$ entre os elementos de maior preocupação ambiental em função de sua maior mobilidade no solo.

\subsection{Adsorção Competitiva}

$\mathrm{Na}$ figura 1 estão representados os dados da adsorção competitiva dos quatro metais traço ao solo amostrado, soluções contendo íons dos quatro metais traço nas mesmas concentrações foram adicionadas ao solo e a adsorção foi investigada, na temperatura de $25,0 \stackrel{\circ}{ } \mathrm{C}$.

Figura 1: Isoterma de adsorção competitiva dos quatro metais traço ao solo amostrado, $25,0^{\circ} \mathrm{C}$

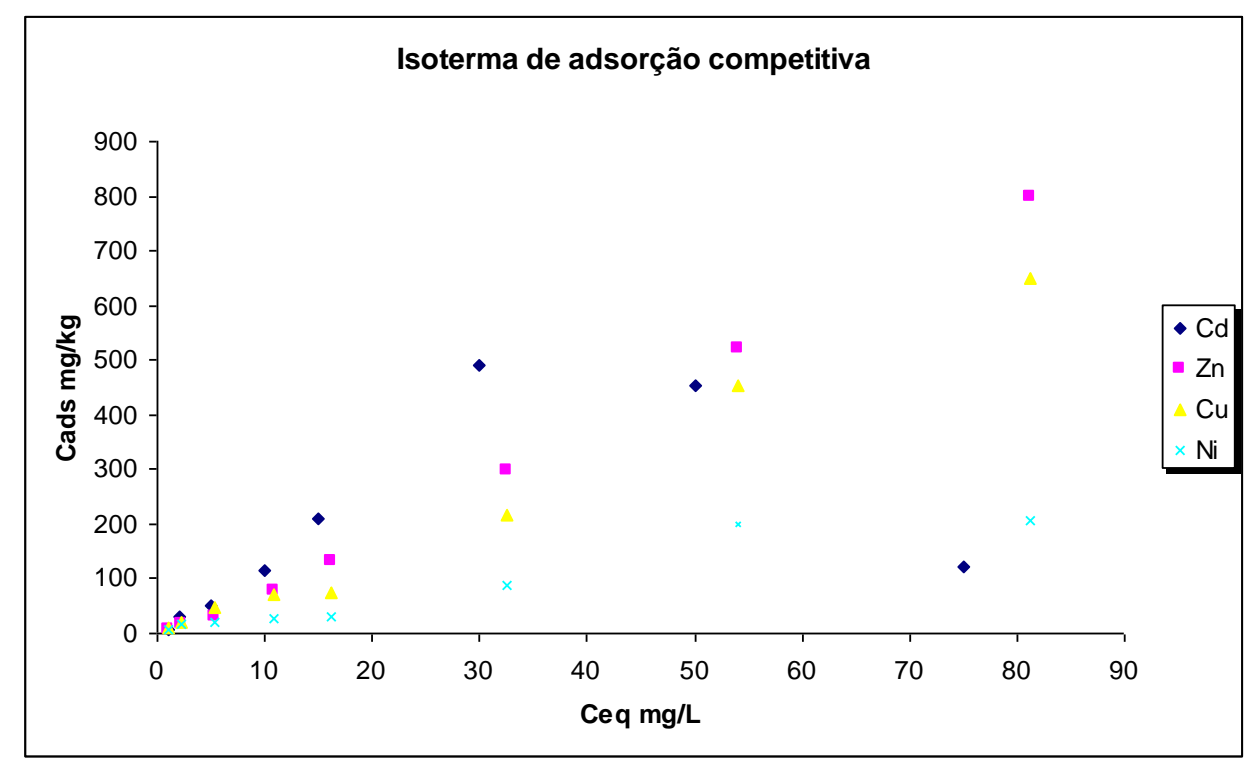

Fonte: Autores, 2014.

Percebe-se que os íons de $\mathrm{Cd}^{+2}$ foram os que apresentaram maior adsorção, seguida da adsorção de íons de $\mathrm{Cu}^{2+}, \mathrm{Zn}^{+2}$ e $\mathrm{Ni}^{+2}$, até cerca da concentração de 30 
$\mathrm{mg} \mathrm{L} \mathrm{L}^{-1}$, a adsorção do $\mathrm{Cd}$ diminui e a do $\mathrm{Ni}$ tende a diminuir após a concentração de $55 \mathrm{mg} \mathrm{L}^{-1}$. A adsorção de $\mathrm{Cu}$, até a concentração de cerca de $5 \mathrm{mg} \mathrm{L}^{-1}$ é maior que do $\mathrm{Zn}$, depois, o comportamento se inverte, as adsorções do $\mathrm{Zn}$ e $\mathrm{Cu}$ continuam crescentes até a maior concentração analisada. Considerando então a adsorção até a concentração de $30 \mathrm{mg} \mathrm{L}^{-1}$, a sequência de adsorção no sistema competitivo, em termos de concentração de adsorção foi: $\mathrm{Cd}>\mathrm{Cu} \geq \mathrm{Zn}>\mathrm{Ni}$. Esta mesma sequência foi observada por Lopes (2009) para três solos do estado de São Paulo classificados em Argissolo vermelho-amarelo com horizonte A tipo moderado e tipo chernozêmico, ambos de textura média e Neossololitólico com horizonte A tipo moderado de textura arenosa. Tal fato também é passível de maiores investigações, pois, fatores como o pH do meio, a formação de hidróxi-compelxos, a presença de óxidos metálicos e ligantes aniônicos, entre outros, influenciam os processos de adsorção. Desta forma, a caracterização do solo quanto aos teores de óxidos de ferro, alumínio e silício se faz necessária além do tamponamento do solo quanto ao $\mathrm{pH}$. Com relação aos ligantes inorgânicos, os experimentos foram realizados em meio de nitrato devido à sua menor habilidade em complexar os cátions metálicos.

\section{CONCLUSÕES}

- As isotermas de Langmuir e Freundlich foram adequadas para descrever os processos de adsorção dos metais traço analisados.

- Observou-se que o metal traço $\mathrm{Cd}$ foi o que apresentou os maiores valores de adsorção máxima (Qmáx), o $\mathrm{Cu}$ apresentou o maior valor da constante de afinidade $(\mathrm{K} \mathrm{L})$ do modelo matemático de Langmuir. $\mathrm{O} \mathrm{Zn}$ apresentou o maior valor de capacidade adsortiva $\left(\mathrm{K}_{\mathrm{f}}\right)$ e o $\mathrm{Cd}$ o maior valor de $1 / \mathrm{n}$ do modelo de Freundlich.

- Considerando o pH natural do solo, a seqüência de afinidade observada foi: $\mathrm{Cu}$ $>\mathrm{Zn}>\mathrm{Ni}>\mathrm{Cd}$, no sistema não competitivo e a seqüência de afinidade $\mathrm{Cd}>\mathrm{Cu} \geq$ $\mathrm{Zn}>\mathrm{Ni}$, no sistema competitivo.

\section{REFERÊNCIAS}

ALLOWAY, B. J. Heavy metals in soils. 2.ed. London: Blackie Academic \& Professional, 1995. 
ANDERSON, P. R.; CHRISTENSEN, T. H. Distribution coefficients of $\mathbf{C d}, \mathbf{C o}, \mathbf{N i}$ and $\mathbf{Z n}$ in soils.J. Soil Science. v.39, p.15-2, 1988.

ANDRADE, J. C.; ABREU, M. F. Análise Química de Resíduos Sólidos para Monitoramento e Estudos Agroambientais. Instituto Agronômico de Campinas (IAC), Campinas (SP), 2006.

ARACRUZ CELULOSE - Uso de defensivos agrícolas.Disponível em: http://www.aracruz.com.br/show arz. Acessado em dezembro de 2014.

ARAúJo, A. L. P.; GIMENES, M. L.; BARROS, M. A. S. D. Scientia Plena. V. 5, n. 12, p. 1-6, 2009.

CONAMA - Conselho Nacional do Meio Ambiente. Resolução № 420/2009. Brasil, 2009.

COONEY, D. O. Adsorption Design for Wastewater Treatment. Boca Raton, Florida: CRC Press, 1999.

DEMUELENAERE, R. G. de A. Caracterização de propriedades de transporte de metais pesados em solos residuais do Rio de Janeiro. Rio, Rio de Janeiro. 2004. Dissertação (Mestrado). Pontifícia Universidade Católica.

FONTES, M. P. F.; GOMES, P. C. Simultaneouscompetitiveadsorptionof heavy metalsbythe mineral matrixoftropicsoils. Applied Geochemistry, v.18, p.795-804, 2003.

FREEZE, R. A.; CHERRY, J. A.Groundwater.New Jersey: Prentice Hall Inc., 1979. 604 p.

GILES, C. H.; MacEWAN, T. H.; NAKHWA, S. N.Studies in adsorptions.Part XI. A system of classification of solution adsorption isotherms, and its use in diagnosis of adsorptions mechanisms and in measurement of specific surface areas of solids.J. Chem. Soc., p.3973-3993, 1960.

GUARACHO, V. V. Remediação Eletrocinética de Chumbo e Níquel em solos de Landfarming de Refinaria.Dissertação(Mestrado), Universidade Federal do Paraná, Curitiba, 2005.

LAIR, G. J. et al. Response of the sorption behavior of $\mathrm{Cu}, \mathrm{Cd}$ and $\mathrm{Zn}$ to different soil management. J. Plant. Nutr. Soil Scie., v. 169, p. 60-68, 2006.

LAVADO, R. S.; PORCELLI, C. A.; ALVAREZ, R. Nutrient and heavy metal concentration and distribution in corn, soybean and wheat as effected by different tillage systems in the Argentine Pampas. Soils\&TillageResearch, v. 62, p. 55-60, 2001.

LOPES, M. C. Adsorção individual e competitiva de $\mathrm{Cd}, \mathrm{Cu}, \mathrm{Zn}$ e $\mathrm{Ni}$ em solos em função da variação do pH. Piracicaba, SP. 2009, 100p. Dissertação (Mestrado) - Escola Superior de Agricultura Luiz de Queiroz.

MACHADO, S. L. et al.Diagnóstico da contaminação por metais pesados em Santo Amaro da Purificação - Bahia. In: Congresso Brasileiro de Geotecnia Ambiental/REGEO' 5. 2003. Porto Alegre. Anais REGEO' 5. Porto Alegre: PUC, 2003. Meio digital.

MOREIRA, C. S.; ALLEONI, L. R. F. Adsorption of $\mathrm{Cd}, \mathrm{Cu}, \mathrm{Ni}$ and $\mathrm{Zn}$ in tropical soils under competitive and non-competitive systems.Sci. Agric., v.67, n.3, p.301-307, 2010.

RUTHVEN, D. M. Principles of Adsorption and Adsorption Processes.New York: John Wiley \& Sons, 433p. 1984. 
SAUVÉ, S; HENDERSHOT, W.; ALLEN, H. E. Solid-solution partitioning of metals in contaminated soils: dependence on pH, total metal burden, and organic matter. Environ. Scie. Tech. V. 34, n. 7, p. $1124-1131,2000$.

SOARES, M. R. Coeficiente de distribuição $\left(K_{d}\right)$ de metais pesados em solos do Estado de São Paulo. Piracicaba, SP, Brasil. 2004, 202 p. Tese (Doutorado) - Universidade de São Paulo.

RAIJ, B. van et al.Análise química para avaliação da fertilidade de solos tropicais.Campinas: InstitutoAgronômico, 2001, 285 p.

SPOSITO, M.The Chemistry of Soils.New York: Oxford University Press, 1989, 277 p.

TOMÉ JR., J. B. Manual para Interpretação de Análise de Solo. Guaíba - Rio Grande do Sul: Agropecuária, 1997, 247p.

UNIVERSIA, Estudo químico dos fertilizantes sintéticos. 2006, disponível em http://www.universia.es/html trad/portada/actualidad/noticia actual. Acessado em dezembro de 2014.

USEPA. Method 3050B Aciddigestionofsediments, sludges, andsoils. 1998.Disponível em: http://www.epa.gov/osw/hazard/testmethods/sw846/pdfs/3050b.pdf. Acessado em fevereiro de 2014.

USEPA - United States Environmental ProtectionAgency. Understanding Variation in Partition Coefficient, $K_{d}$, Values: Review of Geochemistry and Available $K_{d}$ Values for Cadmium, Cesium, Chromium, Lead, Plutonium, Padon, Strontium, Throrium, Tritium (3H), and Uranium. Washington: USEPA, v. 4, 1999. 